\title{
Effect of bronchoscopy on intracranial hypertension during different regimen of sedation by optic nerve sheath diameter
}

\author{
${ }^{1}$ Emine Ozsari, ${ }^{2}$ Abdullah Demirhan \\ ${ }^{1}$ Department of Pulmonology, ${ }^{2}$ Department of Anesthesiology, Bolu Abant Izzet Baysal Univercity \\ Hospital, Turkey
}

\begin{abstract}
Objectives: Fiberoptic bronchoscopy (FOB) is a useful method for ventilator-associated pneumonia (VAP), aspiration, and atelectasis, especially in intensive care units. (ICU) We aimed to investigate the effect of FOB on intracranial pressure by ultrasonographic optic nerve sheath diameter (uONSD) during different sedation protocols in a tertiary ICU. Methods: Prospective randomized study included the patients with two groups as superficial (Group M; midazolam) and deep sedation (Group P; propofol). FOB was performed for VAP or aspiration and intracranial hypertension (ICH) was measured with uONSD, noninvasively. The values of uONSD were noted pre-procedure, on sedation, $1 \mathrm{st}-5 \mathrm{th}$, and 15th minute of the procedure. In addition, mean arterial pressures (MAP), oxygen saturation, and heart rate values were recorded from the monitor. Results: The mean age and indications of FOB for 33 patients as Group M ( $n=17)$ and Group P $(n=16)$ were similar to each other. ONSD was increased with the procedure in both groups but in group $\mathrm{P}$ it was stabilized from the 5 th minutes of FOB whereas became higher progressively in group $M(p<0,001)$. Postoperative MAP values that may have an important role for ICH were also increased in Group M (MAP was $70.65 \pm 16.18$ at time of sedation in Group P and $75.63 \pm 13.76$ in Group M).

Conclusion: This study showed that bronchoscopy results as a significant increase for OSND in both groups but that was less high in patients who had deep sedation with propofol and it returned to baseline after the procedure.
\end{abstract}

Keywords: Bronchoscopy, intensive care unit, optic nerve, propofol

\section{INTRODUCTION}

Fiberoptic bronchoscopy (FOB) is a tool to research for respiratory symptoms such as hemoptysis, wheezing, or coughing. ${ }^{1-4}$ It is also used for both diagnoses of ventilator-associated pneumonia (VAP) and treatment of atelectasis - aspiration, especially in intensive care units (ICU) ${ }^{2,3}$ Although major complications such as hypoxia, arrhythmia, pneumothorax have been reported in the literature, there are few studies showing the effects of FOB on cerebral hemodynamics..$^{5-7}$

Intracranial pressure above $20 \mathrm{mmHg}$ is defined as intracranial hypertension ( $\mathrm{ICH}$ ). Intraventricular and intraparenchymal measurements are the gold standard methods for $\mathrm{ICH} .{ }^{8}$ However, these techniques has limitation in clinical practice because of the risks, such as infection and bleeding. ${ }^{9}$ Ultrasonographic measurement of optic nerve sheath diameter (uONSD) is a new non-invasive method that have been reported to be reliable when compared with invasive techniques.$^{8-10}$ It has been noted that the normal value of ONSD is $4.5 \mathrm{~mm}$, and it indicates intracranial pressure above $20 \mathrm{mmHg}$ when it is measured above $5 \mathrm{~mm} \cdot{ }^{8-10}$ Recent studies examining the effects of FOB was measured invasively using ventriculostomy drain..$^{5-9}$

Volume changes of cerebrospinal fluid (CSF), skull, vascular, parenchymal structures are important for the intracranial pressure (ICP) and some maneuvers like coughing and straining may cause $\mathrm{ICH} .^{9-11}$ Especially, cough increases intrathoracic pressure and central venous pressure (CVP), which affects CSF pressure so using deep sedation can prevent ICH. ${ }^{12}$ High CVP has also been shown to increase ICP through several

Address correspondence to: Emine Ozsari M.D. Bolu Abant Izzet Baysal Univercity Hospital, department of Pulmonology, Turkey. Tel: +903742534656 , email: dreminedemirok@ hotmail.com

Date of Submission: 24 June 2021; Date of Acceptance: 3 July 2021

https://doi.org/10.54029/2021mtd 
mechanisms, such as, decreased venous return to the heart, hypercapnia and respiratory acidosis. ${ }^{11}$ Also, it is known that high airway pressures in mechanical ventilation may cause hemodynamic changes by increasing pleural pressures and causing a functional obstruction in the cerebral venous outlet through the jugular venous system. ${ }^{13}$ In this study, we aimed to identify a regimen of anesthesia and sedation that could confer protection against increases in ICP during FOB by uOSND in ICU, noninvasively.

\section{METHODS}

Between March 2018 and July 2019, 33 patients needed FOB for diagnosis of VAP or aspiration in an ICU, a tertiary hospital. Patients with a history of head trauma, intracranial surgery, intracranial mass, using corticosteroid treatment, having bleeding diathesis, hemoglobin levels less than $10 \mathrm{mg} / \mathrm{dl}$, pregnant women, and patients under 18 years of age were excluded from the study. And if patients in continuous sedation need extra doses, they were excluded too. This randomized prospective study was conducted in accordance with the Helsinki Declaration and with the permission of the Abant Izzet Baysal University Hospital ethics committee (Local Ethics Number: 2018/69). Written informed consent was obtained from first-degree relatives of all patients.

\section{Anesthesia}

All patients were intubated in ICU. Volumecontrolled ventilation modes were preferred during FOB. The inspired $\mathrm{FiO} 2$ was raised to $100 \%$ before the procedure. Mean blood pressure, heart rate, oxygen saturation, and blood gas values were closely monitored during the procedure. Enteral feeding was stopped at least 6 hours before the procedure. The endotracheal tube position was checked at the end of the procedure. FOB was performed using a mobile bronchoscopy (Storz FB; $5.0 \mathrm{~mm}$ outer diameter). Patients were randomized into two groups by an anesthetist specialist who was blinded to the study. Patients in Group M received i.v. 0:02 to $0: 04 \mathrm{mg} / \mathrm{kg}$ midazolam (zolamid $15 \mathrm{mg} / 3$ $\mathrm{mL}$, and medications, Turkey), whereas patients in Group P received propofol i.v. 1.5 to $2.5 \mathrm{mg} /$ $\mathrm{kg}(1 \%$ propofol, Fresenius, Turkey) were given. Neither any of the opioids such as fentanyl, remifentanil nor the muscle relaxants were used before the procedure.

\section{Optic nerve sheath diameter}

ONSD was performed with the B-Mode setting with the 6-10 MHz linear probe of the ultrasound device (L38/10-5 Mhz Transducer, SonoSite, Inc. Bothell, WA98021USA.). During measurements, the patients were placed in the supine position and the eyelid was closed with stretch film to protect the eyeball while the eyelids were closed. All ONSD measurements were performed by a single researcher experienced in the use of ultrasound who was blinded to the study. The transverse image of the eyeball was obtained in the axial plane without pressure on the eyeball using the high-frequency linear transducer. The best image was obtained for the optic nerves of both eyes and the image was frozen and the cursor was placed $3 \mathrm{~mm}$ posterior from the papilla to the external contours of the optic nerve and the measurement was completed. All values greater than $5.0 \mathrm{~mm}$ were evaluated as an ICH. The ONSD was measured by ultrasonography pre-procedure (T1), on sedation (T2), 1st minute (T3) - 5th minute (T4), and 15th minute (T5) of the procedure.

\section{Statistical analysis}

Univariate comparisons were made using various statistical tests. The normality of continuous variables was evaluated by Shapiro-Wilk tests. Variables showing normal distribution were analyzed by independent samples t-tests. MannWhitney U tests were used for continuous variables without normal distribution. Bonferonni corrected paired sample t-tests were used to compare the variables between the two groups. Categorical variables were analyzed by Chisquare test, and Fisher's exact correction was performed when necessary. After adjusting age, sex and BMI, multivariate repeated measures ANOVA was performed to see the effect of time and drug. Significance was set at $p<0.05$ and analyzes were performed using the Social Sciences 25.0 Statistical Package for Windows (SPSS Inc., Chicago, Illinois, USA).

\section{RESULTS}

Thirty-three patients, 17 female, and 16 male were included in two groups as Group $M(n=17)$ and Group P $(n=16)$ for this study. Bronchoscopy was performed on patients for diagnosis of VAP or aspiration. In both groups, mean age (Group M: 76.94 \pm 11.51 ; Group P: $68.75 \pm 19.30$ ) and duration of the procedure (15 minutes) were similar. Also, physiological changes between the groups for 
arterial blood gas $(\mathrm{pH}, \mathrm{PCO} 2, \mathrm{PO} 2, \mathrm{SpO} 2$, and lactate values) before and after the procedure were similar too. However, in Group M, heart rate and MAP values were elevated at all times of periods during the procedure. (Table 1) Repeated measurements according to the GreenhouseGeisser corrected ANOVA assessment, there was a statistically significant difference between the time points of the ONSD. (F $(2.957,82.805)$ $=0.880, \mathrm{p}=0.030)$. As a result of post hoc tests using Bonferroni correction, significant differences were observed between T2-T3, T2-T4, and T2-T5 time frames in Group P. The ONSD of the midazolam group has significant increases between all the time periods. $(\mathrm{p}<0.005)$ (Table 2$)$ Also the level of ICP in Group P stayed on the

Table 1: Clinical and numerical values of physiological parameters observed during the procedure

\begin{tabular}{|c|c|c|}
\hline & Group M & Group P \\
\hline Number of patients & 17 & 16 \\
\hline Mean age & $76.94 \pm 11.51$ & $68.75 \pm 19.30$ \\
\hline \multicolumn{3}{|l|}{ Indication of FOB } \\
\hline VAP & 7 & 9 \\
\hline Aspiration & 10 & 7 \\
\hline \multicolumn{3}{|c|}{ Mean arterial pressure (MAP) } \\
\hline Pre-p & $87.06 \pm 11.95$ & $84.71 \pm 16.37$ \\
\hline Sed & $75.63 \pm 13.76$ & $70.65 \pm 16.18$ \\
\hline $1 \mathrm{st} \min$ & $91.06 \pm 18.38$ & $81.53 \pm 25.94$ \\
\hline 5 th $\min$ & $86.38 \pm 12.48$ & $83.35 \pm 18.75$ \\
\hline 15 th $\min$ & $76.75 \pm 14.19$ & $72.65 \pm 13.1$ \\
\hline \multicolumn{3}{|l|}{ Heart rate (HR) } \\
\hline Pre-p & $106.71 \pm 25.57$ & $95.69 \pm 15.86$ \\
\hline Sed & $106.29 \pm 26.49$ & $94.38 \pm 22.91$ \\
\hline $1 \mathrm{st} \min$ & $113.59 \pm 30.42$ & $101.63 \pm 27.77$ \\
\hline 5 th $\min$ & $114.53 \pm 31.54$ & $100.44 \pm 29.53$ \\
\hline 15 th $\min$ & $115.82 \pm 30.53$ & $96.25 \pm 24.23$ \\
\hline \multicolumn{3}{|l|}{ Blood gases } \\
\hline Ph Pre $-\mathrm{p}$ & $7.41 \pm 0.06$ & $7.44 \pm 0.09$ \\
\hline 15 th $\min$ & $7.39 \pm 0.07$ & $7.45 \pm 0.08$ \\
\hline pC02 pre - p & $47.47 \pm 10.92$ & $39.94 \pm 7.78$ \\
\hline 15 th $\min$ & $49.35 \pm 11.41$ & $39.81 \pm 7.88$ \\
\hline P02 pre - p & $66.12 \pm 19.76$ & $70.31 \pm 20.76$ \\
\hline 15 th $\min$ & $74.12 \pm 22.15$ & $79.63 \pm 20.81$ \\
\hline Sp02 pre $-\mathrm{p}$ & $91.82 \pm 9.17$ & $95.25 \pm 6.56$ \\
\hline 15 th $\min$ & $94.41 \pm 6.71$ & $95.44 \pm 7.8$ \\
\hline Lact pre $-p$ & $1.35 \pm 0.37$ & $1.25 \pm 0.54$ \\
\hline 15 th $\min$ & $1.25 \pm 0.37$ & $1.43 \pm 0.44$ \\
\hline \multicolumn{3}{|l|}{ ONSD values } \\
\hline Pre-p & $4.5 \pm 0.09$ & $4.8 \pm 0.12$ \\
\hline Sed & $4.4 \pm 0.08$ & $4.4 \pm 0.08$ \\
\hline 1 st $\min$ & $4.9 \pm 0.10$ & $4.8 \pm 0.07$ \\
\hline 5 th $\min$ & $5.3 \pm 0.07$ & $5.1 \pm 0.06$ \\
\hline 15 th $\min$ & $5.4 \pm 0.08$ & $5.1 \pm 0.07$ \\
\hline
\end{tabular}

Data expressed as mean \pm SE. Paired samples t-tests were used. (lact: lactate, Sp 02: oxygen saturation, p02: oxygen pressure, pCo2: carbon dioxide pressure ONSD : optic nerve sheath diameter) (PreP : T1, sed : T2, 1st min : T3, 5th $\min : \mathrm{T} 4,15$ th $\min : \mathrm{T} 5)$ 
Table 2: Comparison of ONSD measurements between groups with time points

\begin{tabular}{lll}
\hline & Group $\mathbf{M}(\mathbf{n}=\mathbf{1 7})$ & Group P $(\mathbf{n}=\mathbf{1 6})$ \\
\hline T1-T2 & 0.123 & 0.213 \\
T1-T3 & 0.098 & 0.755 \\
T1-T4 & $<\mathbf{0 . 0 0 1}$ & 0.419 \\
T1-T5 & $<\mathbf{0 . 0 0 1}$ & 0.454 \\
T2-T3 & 0.028 & $\mathbf{0 . 0 0 4}$ \\
T2-T4 & $\mathbf{< 0 . 0 0 1}$ & $\mathbf{< 0 . 0 0 1}$ \\
T2-T5 & $<\mathbf{0 . 0 0 1}$ & $\mathbf{0 . 0 0 3}$ \\
T3-T4 & 0.042 & 0.093 \\
T3-T5 & 0.021 & 0.181 \\
T4-T5 & 0.540 & 0.937 \\
\hline
\end{tabular}

p-values obtained with paired samplest-tests. The bold p-values indicate statistical significance with Bonferroni correction at $\alpha / 10=0.005$.

( PreP : T1, sed : T2, 1st $\min :$ T3, 5th $\min :$ T4, 15th min : T5 )

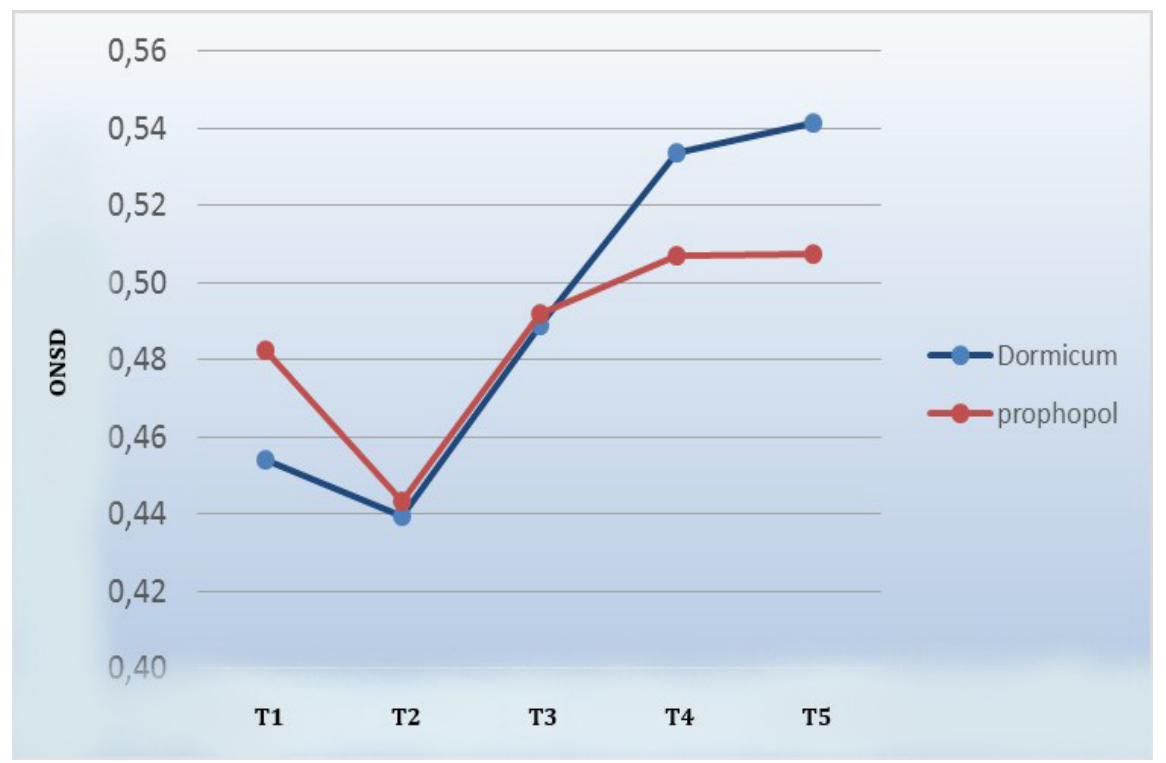

Figure 1. Measurements of ONSD between time intervals of two groups

same line during T3, T4, and T5 while ICP had been increasing all of the periods in Group M. (Figure 1). No neurological complications were observed in the postoperative period.

\section{DISCUSSION}

In our study, we evaluated the effects of bronchoscopy on ICP during different regimens of sedation with ultrasound-guided OSND before and after procedure non-invasively. All patients were divided into two groups as superficial (Group M) and deep (Group P) sedation. When a significant increase of OSND was observed between all time periods in Group $\mathrm{M}$, there was a less increase and a parallel course after the 5th minute in Group P.
Also we found that this increase was nearly the same as pre-procedure in group $\mathrm{P}$.

Bronchoscopy is considered to be technically safe, but it is not completely uncomplicated. It has some risks of hypoxemia, hypercarbia, and respiratory failure, especially secondary to airway obstruction. Also, it may cause various complications such as arrhythmia, bleeding, hypotension/hypertension, pneumothorax, and ICH. ${ }^{3-7}$

Several factors that may lead to an increase in ICP during FOB should be considered. First of all, the position of the patient's head is important because ICP may be affected by resistance to venous outflow. ${ }^{11}$ Second, the neck is extended to maximize the patient's airway patency especially 
critical for cerebral perfusion pressure in severe trauma. ${ }^{12,14}$ Also while small changes in circulating volume within physiological limits may not cause pressure changes, ICP may change even with a minor increase especially in patients with trauma in ICU. ${ }^{12}$ So it is recommended to avoid FOB, especially in the first 24 hours in such patients. Baiwa et $a l .{ }^{15}$ examined patients with cranial trauma who had both FOB and cranial computed tomography and found increased intracranial pressure findings on $\mathrm{CT}$ in 29 patients after $\mathrm{FOB}$, retrospectively. They reported when patients were sedated with meperidine and atropine, there was no evidence of neurological complications was observed after 6 weeks post-procedure. In our study, ONSD was $4.9 \pm 0.100 \mathrm{~mm}$ in Group M, while it was $4.8 \pm 0.070 \mathrm{~mm}$ in Group $\mathrm{P}$ on $1 \mathrm{st}$ minute of the procedure(T3). And ONSD was stabilized on the 5th and 15th minutes of FOB in Group $\mathrm{P}$ whereas it became higher progressively in Group M $(\mathrm{p}<0.001)$.

In severe head trauma, it was concluded that high ICH may be balanced with the increase in MAP and sufficient CPP was maintained. Peerles et $a l .{ }^{16}$ anesthetized fifteen patients that had a brain injury, in need of FOB with thiopental and lidocaine and examined the values of ICP, MAP, and cerebral perfusion pressure $(\mathrm{CCP})$ before and after the procedure with intracranial monitoring. They found an average increase in ICP after FOB $13.5 \mathrm{~mm} \mathrm{Hg}$ above basal values. However, ICP has been shown to return to basal levels after treatment. In their study, the mean increase in MAP was 92.3: $\pm: 16.1 \mathrm{~mm} \mathrm{Hg}$ to $111.5 \pm 13.9$ $\mathrm{mmHg}$ when ICP was highest. Similarly, in our study, MAP had increased from the beginning of the procedure in both groups. However, in Group P, the rises of the MAP values were less high than in group M. MAP was $70.65 \pm 16.18$ on sedation(T2) in Group P and $75.63 \pm 13.76$ in Group M. This was thought to be related to the depth of anesthesia. Kerwin et al. ${ }^{7}$ used vecuronium, morphine sulfate, and midazolam as standard anesthetic regimens to prevent ICP increase during FOB, but they could not provide sufficient sedation or analgesia. These studies have shown that optimal sedation is required to reduce intracranial response during FOB and better comfort is achieved by the addition of muscle relaxants to the standard regimen.

There are much data in the literature about the effects of tracheostomy and endotracheal maneuvers on ICP. Some reasons for the ICH by endotracheal suctioning may be the stimulation of the airways, which may be due to the cough reflex, as well as to the endotracheal tube and PEEP. ${ }^{18-22}$ These procedures have been suggested that should be performed with anti-edema treatment in patients in ICU. It has emphasized that the need to determine appropriate analgesia and sedation regimens in order to reduce this effect. ${ }^{6,17}$ In a recent study, tracheostomy was performed in a group of patients at risk for ICP in neurology ICU. In that study, atropine, propofol, fentanyl, and atracurium were used and anti-edema treatment was started when the ICP exceeded $30 \mathrm{mmHg} .{ }^{17}$ A recent study with endotracheal aspiration, lidocaine $(1.5 \mathrm{mg} / \mathrm{kg})$ before in 10 patients receiving head trauma anti-edema treatment and compared the results with placebo group given saline. ${ }^{23}$ They found that although ICP increased above $20 \mathrm{~mm} \mathrm{Hg}$ in all patients, the increase in ICP in lidocaine-treated group was lower than the increase in the placebo-treated group. To prevent ICP increase during intubation, White $e t$ $a l .{ }^{24}$ used i.v. fentanyl, lidocaine, thiopental. They suggested that neuromuscular blockers used in combination with topical anesthetics may be the most effective combination to prevent increases in ICP associated with endotracheal aspiration.

In our study, we performed routine sedation for FOB. We determined our sedation application deeply and superficially and researched the changes in ONSD. In patients who were given propofol and increased depth of anesthesia, fewer reflexes such as retching and straining during the procedure and a more stable HR value of the patients led to a less increase in the ONSD, thus reducing the increase in intracranial pressure.

There are some limitations of our study. Although evidence of persistent intracranial hypertension after FOB has not been demonstrated, we did not record the Glasgow coma score. Second of all, other factors influencing ICP like airway pressures, ETCO2 during bronchoscopy can be considered. Further studies are necessary with a large number of patients to identify ICP changes, especially in the population.

In conclusion, we suggest that patients who performed bronchoscopy with propofol had a lesser increase and a stable line in ONSD when compared to those receiving midazolam in the ICU population. As emphasized in our study, a regimen of anesthesia and sedation can be preferred for protection against increases in ICP during FOB measuring the diameter of optic nerve sheath by ultrasonographic method non-invasively. 


\section{DISCLOSURE}

Availability of data and material: The datasets used and/or analysed during the current study are available from the corresponding author upon reasonable request.

Financial support: None

Conflicts of interest: There were no conflicts of interest in this study.

\section{REFERENCES}

1. Jin F, Mu D, Chu D, Fu E, Xie Y, Liu T. Severe complications of bronchoscopy. Respiration 2008;76:429-33.

2. Eapen GA, Shah AM, Lei X, Jimenez CA, Morice RC, Yarmus L. Complications, consequences, and practice patterns of endobronchial ultrasound-guided transbronchial needle aspiration: Results of the AQuIRE registry. Chest 2013;143:1044-53.

3. Schnabel RM, van der Velden K, Osinski A, Rohde G, Roekaerts PM, Bergmans DC. Clinical course and complications following diagnostic bronchoalveolar lavage in critically ill mechanically ventilated patients. BMC Pulm Med 2015;15:107.

4. Facciolongo N, Patelli M, Gasparini S, Lazzari Agli $\mathrm{L}$, Salio M, Simonassi C. Incidence of complications in bronchoscopy. Monaldi Arch Chest Dis 2009;71:814.

5. Lee TS. Fiberoptic bronchoscopy and intracranial pressure. Chest 1994;105:1909.

6. Kleffmann J, Pahl R, Deinsberger W, Ferbert A, Roth C. Effect of percutaneous tracheostomy on intracerebral pressure and perfusion pressure in patients with acute cerebral dysfunction. Neurocrit Care 2012;17:85-9.

7. Kerwin AJ, Croce MA, Timmons SD, Maxwell RA, Malhotra AK, Fabian TC. Effects of fiberoptic bronchoscopy on intracranial pressure in patients with brain injury. J Trauma 2000;48:87882;82-3.

8. Kimberly HH, Shah S, Marill K, Noble V. Correlation of optic nerve sheath diameter with direct measurement of intracranial pressure. Acad Emerg Med 2008;15:201-4.

9. Geeraerts T, Merceron S, Benhamou D, Vigue B, Duranteau J. Non-invasive assessment of intracranial pressure using ocular sonography in neurocritical care patients. Intensive Care Med 2008;34:2062-7.

10. Dubourg J, Javouhey E, Geeraerts T, Messerer M, Kassai B. Ultrasonography of optic nerve sheath diameter for detection of raised intracranial pressure. Intensive Care Med 2011;37:105968.

11. Bloomfield GL, Ridings PC, Blocher CR, Marmarou A, Sugerman HJ. A proposed relationship between increased intra-abdominal, intrathoracic, and intracranial pressure. Crit Care Med 1997;25:496503.

12. Ben-Haim M, Mandeli J, Friedman RL, Rosenthal RJ. Mechanisms of systemic hypertension during acute elevation of intraabdominal pressure. J Surg
Res 2000;91:101-5.

13. Winklewski PJ, Wolf J, Gruszecki M, WszedybylWinklewska M, Narkiewicz K. Current understanding of the effects of inspiratory resistance on the interactions between systemic blood pressure, cerebral perfusion, intracranial pressure, and cerebrospinal fluid dynamics. J Appl Physiol 2019;127:1206-14.

14. Metzger A, Rees J, Kwon Y, Matsuura T, McKnite S, Lurie KG. Intrathoracic pressure regulation improves cerebral perfusion and cerebral blood flow in a porcine model of brain injury. Shock 2015;44(Suppl 1):96102 .

15. Bajwa MK, Henein S, Kamholz SL. Fiberoptic bronchoscopy in the presence of space-occupying intracranial lesions. Chest 1993;104:101-3.

16. Peerless JR, Snow N, Likavec MJ, Pinchak AC, Malangoni MA. The effect of fiberoptic bronchoscopy on cerebral hemodynamics in patients with severe head injury. Chest 1995;108:962- 5.

17. Kocaeli H, Korfali E, Taskapilioglu O, Ozcan T. Analysis of intracranial pressure changes during early versus late percutaneous tracheostomy in a neurointensive care unit. Acta Neurochir 2008;150:1263-7; discussion 1267.

18. Rudy EB, Baun M, Stone K, Turner B. The relationship between endotracheal suctioning and changes in intracranial pressure: a review of the literature. Heart Lung 1986;15:488-94.

19. Rudy EB, Turner BS, Baun M, Stone KS, Brucia J. Endotracheal suctioning in adults with head injury. Heart Lung 1991;20:66774.

20. Parsons LC, Shogan JS. The effects of the endotracheal tube suctioning/manual hyperventilation procedure on patients with severe closed head injuries. Heart Lung 1984;13:372-80.

21. McGuire G, Crossley D, Richards J, Wong D. Effects of varying levels of positive end-expiratory pressure on intracranial pressure and cerebral perfusion pressure. Crit Care Med 1997;25:1059-62.

22. Previgliano IJ, Ripoll PI, Chiappero G, et al. Optimizing cerebral perfusion pressure during fiberoptic bronchoscopy in severe head injury. Acta Neurochir Suppl 2002;81:103-5.

23. Donegan MF, Bedford RF. Intravenously administered lidocaine prevents intracranial hypertension during endotracheal suctioning. Anesthesiology 1980;52:516-8.

24. White PF, Schlobohm RM, Pitts LH, Lindauer JM. A randomized study of drugs for preventing increases in intracranial pressure during endotracheal suctioning. Anesthesiology 1982;57:24. 